\title{
Synthesis of gold micro- and nano-wires by infiltration and thermolysis
}

\author{
E.A. Olevsky, ${ }^{\mathrm{a}}$ X. Wang, ${ }^{\mathrm{a}}$ E. Bruce, ${ }^{\mathrm{a}}$ M.B. Stern, ${ }^{\mathrm{b}}$ S. Wildhack ${ }^{\mathrm{c}}$ and F. Aldinger ${ }^{\mathrm{c}}$ \\ ${ }^{a}$ College of Engineering, San Diego State University, San Diego, CA 92182, USA \\ ${ }^{h}$ Sun Microsystems Inc., CTO Physical Sciences Center, San Diego, CA 92121, USA \\ ${ }^{c}$ Max-Planck-Institute for Metal Research, Stuttgart D-70569, Germany
}

\begin{abstract}
An approach for synthesizing micro- and nano-sized gold wires by infiltration and thermolysis is investigated. A porous $\mathrm{ZrO}_{2}$ ceramic preform with aligned pores obtained by unidirectional freezing and freeze-drying is employed as an infiltration template. The sintered porous $\mathrm{ZrO}_{2}$ preform is then infiltrated by a brushing gold solution. The thermolysis is conducted at $600{ }^{\circ} \mathrm{C}$ in air. Micro- and nano-sized gold wires are developed within the walls of the pores after thermolysis. The diameter of the gold wires ranges from several hundred nanometers to several microns.
\end{abstract}

Ultrafine and nano-sized wires and rods are drawing interest due to their unique electrical, mechanical and optical properties. Several approaches, such as vapor phase routing, solution-based methods and templatedirected synthesis, have been utilized to synthesize nano-wires [1]. The template method is a straightforward approach pioneered by Martin [2]. In this technique, a porous alumina membrane with nano-sized pores provided a template for the production of nano-wires when the pores were infiltrated by gold. The templates used in this procedure are either polymer films containing track-etched channels or alumina films containing anodically etched pores [1]. One of the most important benefits of the template method is that the diameter of the nanowires can be controlled at will by varying the diameter of the pores in the template membrane [3]. By depositing two materials into the template simultaneously, composite nano-wires can be synthesized [4]. Goring et al. [5] developed a simple one-step procedure for the synthesis of carbon/gold wires/fibers by the impregnation of gold solutions followed by annealing.

We explored a new approach for the synthesis of micro- and nano-sized gold wires using a $\mathrm{ZrO}_{2}$ porous template. The porous $\mathrm{ZrO}_{2}$ was obtained by unidirec- tional freezing followed by freeze-drying. Freeze-drying was utilized to obtain $\mathrm{Si}_{3} \mathrm{~N}_{4}$ and $\mathrm{Al}_{2} \mathrm{O}_{3}$ preforms with aligned channels by Fukasawa et al. [6,7]. By controlling the temperature gradient in the crystallization process within a powder slurry, this approach provides a convenient method for the fabrication of ceramic preforms with controlled pore morphology. Generally, this methodology does not involve chemical reactions; therefore, it is applicable to any material as long as it is in the form of powder. By controlling the solidification parameters, such as the chemical composition of the slurry, temperature gradient and solidification rate, the structure can be controlled. Our research indicates that it is promising to use preforms with aligned channels as the templates for gold wire growth. It should also be noted, that, besides the potential usage as components of electronic circuitry, gold wires of broad diameter range can be utilized in biochemical sensors.

Our experiments included the zirconia slurry preparation, unidirectional freezing and freeze-drying, sintering of zirconia preforms, infiltration of brushing gold and thermolysis. The details of the experimental procedures are described below.

A 35 vol.\% concentration slurry was made by dispersing $\mathrm{ZrO}_{2}$ powders (TZ-3YS Tosoh, Japan, specific surface area $\left.6.6 \mathrm{~m} \mathrm{~g}^{2} \mathrm{~g}^{-1}\right)$ in deionized water. Commercial dispersant (Duramax D-3005, Rohm and Hass, USA) was added at the ratio of $2.2 \mathrm{mg} / \mathrm{m}^{-2}$ of total particle 
surface area. The slurry was milled in a planetary ball mill (Fritsch LC-106A) for 30 min prior to freezing. The slurry had been further deaerated in a vacuum chamber before freezing. After the above-mentioned processing, the slurry was stable and homogeneous based on visual inspection.

The $\mathrm{ZrO}_{2}$ preforms with aligned channels used in this work were prepared largely in accordance with a process pioneered by Fukasawa et al. [6], and are summarized only briefly here. The unidirectional freezing configuration is similar with that of Fukasawa except that liquid nitrogen replaces liquid ethanol. The configuration is shown in Figure 1. The diameter of the cylindrical mold is $12.5 \mathrm{~mm}$ and the height is $12.5 \mathrm{~mm}$. The $\mathrm{ZrO}_{2}$ slurry was smoothly poured into the mold. Then the bottom part of the aluminum rod was dipped into liquid nitrogen. After the entire slurry was frozen, it was removed from the mold and dried in a freeze dryer (Labconco FreeZone) for $24 \mathrm{~h}$ to sublimate the ice.

Dried samples were sintered in a tube furnace in air at $1400{ }^{\circ} \mathrm{C}$ for $2 \mathrm{~h}$. The heating rate was $10^{\circ} \mathrm{C} \mathrm{min}{ }^{-1}$. After sintering, the preforms were cooled down to room temperature at the rate of $10{ }^{\circ} \mathrm{C} \mathrm{min}$. The sintered $\mathrm{ZrO}_{2}$ preforms were cut perpendicular to the axis into $2 \mathrm{~mm}$ thick slices for brushing gold infiltration.

The $\mathrm{ZrO}_{2}$ preforms were dipped into a brushing gold solution (Alfa Aesar, USA) for infiltration. After that, they were dried in air for $1 \mathrm{~h}$. The dried samples were annealed in a furnace (Ney 2-1350, USA) in air at $600{ }^{\circ} \mathrm{C}$ for $1 \mathrm{~h}$. The heating rate was $5{ }^{\circ} \mathrm{C} \mathrm{min}^{-1}$. When the samples were completely cooled down to room temperature, they were dipped into the brushing gold again for the second infiltration. This process was repeated four times in order to increase the yield of gold wires. After annealing, the samples have been characterized by scanning electron microscopy (SEM).

The sintered $\mathrm{ZrO}_{2}$ contains aligned channels parallel to the direction of the solidification of the ice. Figure 2 shows the sintered preform with aligned porosity. The sintered preform is highly porous because of the presence of both aligned channels (Fig. 2a) and smallscale pores in a zirconia matrix (Fig. 2b). The aligned pores are opened, extending along the direction of solidification. The open porosity enables the infiltration of brushing gold and provides the template for growth of gold wires. The unidirectional solidification was generated by the temperature gradient in the slurry. As a

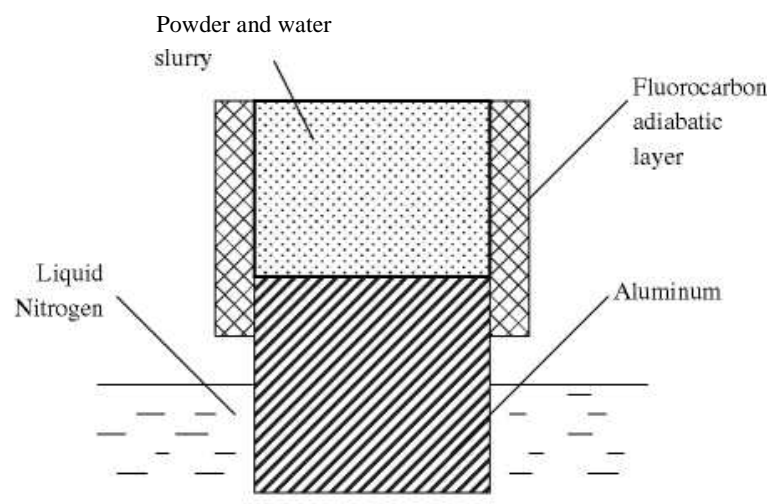

Figure 1. Unidirectional freezing of powder slurry.
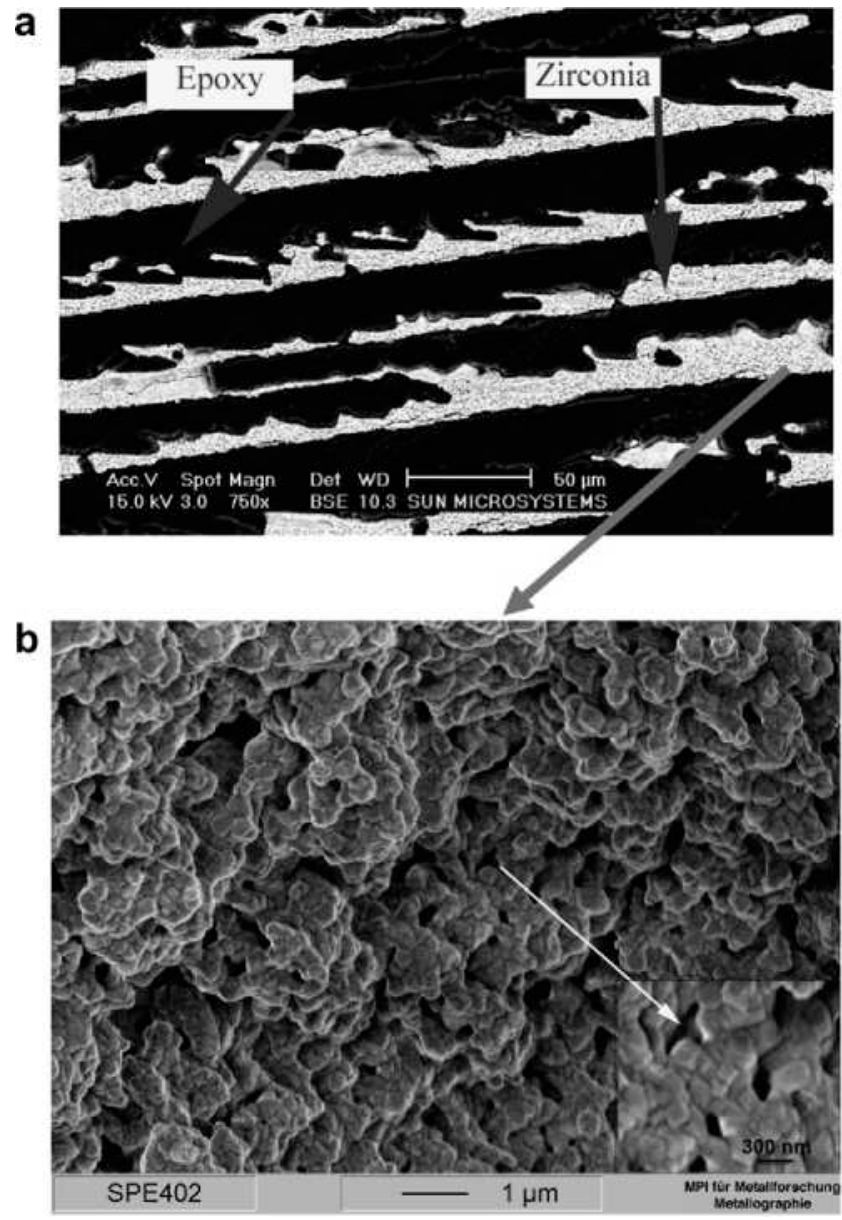

Figure 2. Sintered $\mathrm{ZrO}_{2}$ preform.

result, the ice dendrites grew along the direction of the temperature gradient. Since $\mathrm{ZrO}_{2}$ particles are insoluble in water, they are repelled from the ice dendrites and trapped in between the columnar ice dendrites to form the aligned porous structure. It can be seen from Figure 2 that there are some protruded ceramic agglomerates on the walls of the pores. Apparently, these ceramic agglomerates have been formed because they were trapped by the second dendrite arms during freezing.

A sintered $\mathrm{ZrO}_{2}$ sample frozen in a regular freezer without a directional temperature gradient has a randomly distributed pore structure which indicates the lack of oriented crystal growth due to the absence of the temperature gradient during freezing.

The sintered $\mathrm{ZrO}_{2}$ preforms have been dipped into the brushing gold for infiltration. Gold wires were found to grow in the pores of the preform, as indicated in Figure 3a. A large fraction of the volume of the pores is still empty; the gold wires occupy only a portion of the pore space. Some of the gold wires are straight (Fig. 3a); some of them are bent (Fig. 3b). The presence of bent gold wires indicates their ductility. The diameter of the wires varies from several tens of nanometers to several microns. The maximum length of the wires is larger than $50 \mu \mathrm{m}$. The recrystallization conditions may influence the diameter of the wires. The micrographs also indicate that the diameter of each wire is uniform throughout its length. The energy dispersive 

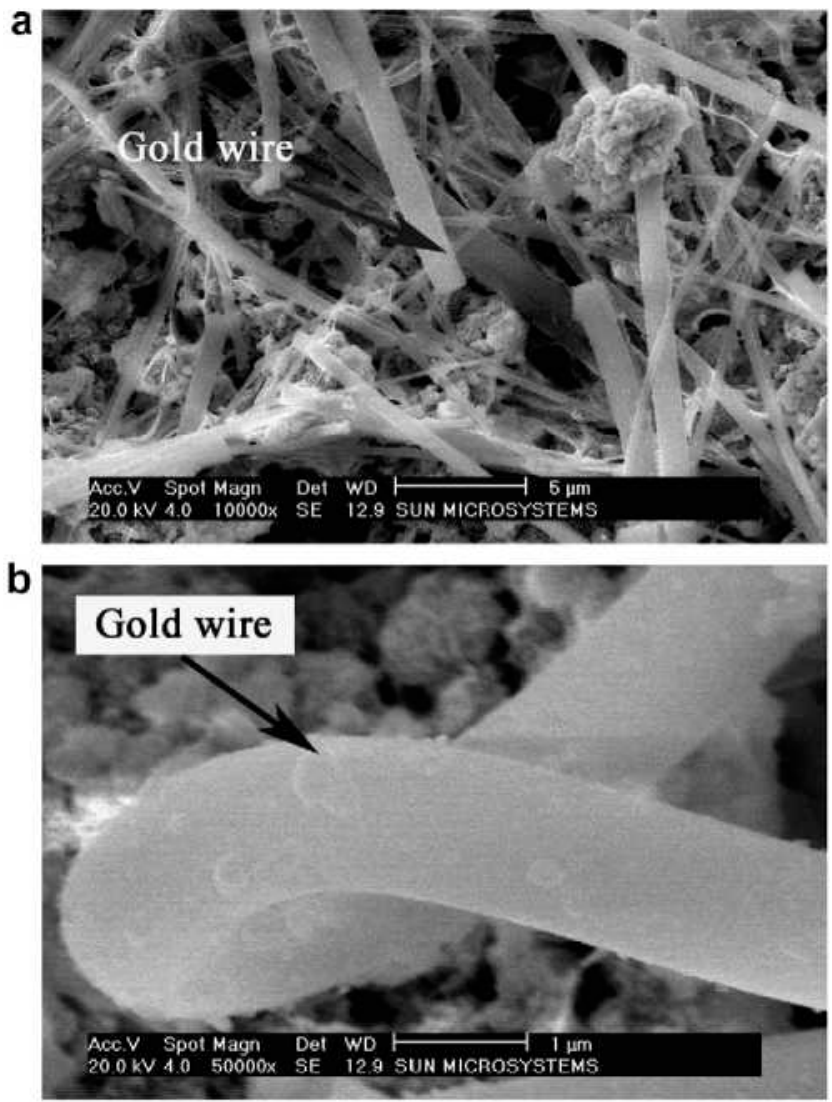

Figure 3. Micrograph of gold wires.

X-ray (EDX) results confirmed the composition of the obtained gold wires. The results of the EDX analysis of the surrounding $\mathrm{ZrO}_{2}$ preform have also been recorded.

The large-scale pore diameters obtained in the experiments are in the ballpark of $\sim 25 \mu \mathrm{m}$, which is much larger than the size of the gold wires. This means that the gold wires did not replicate the pore size and morphology. Fukasawa et al. [6] found the growth of fibrous $\mathrm{Si}_{3} \mathrm{~N}_{4}$ grains in the aligned channels after sintering. It was speculated that the fibrous grains were formed by the evaporation-condensation mechanism.

In this work, the gold wires are formed during the annealing process while the recrystallization occurs. It appears that during the recrystallization process, the wetting conditions between the brushing gold and the $\mathrm{ZrO}_{2}$ preform have substantial impact on the driving force of the growth of the gold wires. A detailed analysis of the thermodynamics and kinetics of this gold wire growth is beyond the scope of this introductory research effort and is a promising subject of further investigations.

In order to identify the influence of the template on the gold wire growth, another comparative experiment has been conducted by placing brush gold on the surface of a transparent glass substrate. The same annealing

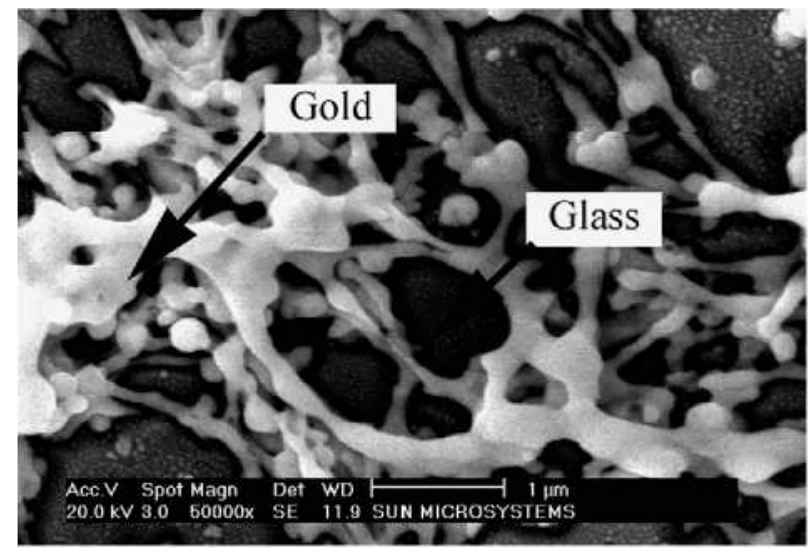

Figure 4. Brush gold annealing on glass.

procedure as the one used for the porous $\mathrm{ZrO}_{2}$ preforms was applied. A micrograph of the annealed structure is shown in Figure 4. From Figure 4, it is clear that there are no gold wires (fibers) present. The gold wire network forms only during the annealing process within the zirconia porous preform.

Thus, as a result of the present work, $\mathrm{ZrO}_{2}$ preforms with aligned pores were synthesized by unidirectional freezing and freeze-drying. After sintering, the preforms were infiltrated by brushing gold. The brushing gold within the $\mathrm{ZrO}_{2}$ preforms was annealed at $600{ }^{\circ} \mathrm{C}$ in air. Gold wires with diameters ranging from several tens of nanometers to several microns were found to grow within the walls of the porous preform. The comparative study of the annealing of the brush gold solution conducted both within $\mathrm{ZrO}_{2}$ preforms and on a flat glass substrate indicates that the unique structure of the $\mathrm{ZrO}_{2}$ preform provides the required conditions for the gold wire growth.

The support of the Alexander-von-Humboldt Foundation is gratefully acknowledged. The support of the National Science Foundation, Division of Manufacturing and Industrial Innovations (Grant DMI0354857) is gratefully acknowledged.

[1] Y.N. Xia, P.D. Yang, Y.G. Sun, Y.Y. Wu, B. Mayers, B. Gates, Y.D. Yin, F. Kim, Y.Q. Yan, Adv. Mater. 15 (5) (2003) 351.

[2] C.R. Martin, Science 266 (1994) 1961.

[3] S. Yu, N. Li, J. Wharton, C.R. Martin, Nano Lett. 3 (6) (2003) 815.

[4] J. Zhang, G. Shi, C. Liu, L. Qu, M. Fu, F. Chen, J. Mater. Sci. 38 (2003) 2423.

[5] P. Goring, E. Pippel, H. Hofmeister, R.B. Wehrspohn, M. Steinhart, U. Gosele, Nano Lett. 4 (6) (2004) 1121.

[6] T. Fukasawa, Z. Deng, M. Ando, T. Ohji, S. Kanzaki, J. Am. Ceram. Soc. 85 (9) (2002) 2151.

[7] T. Fukasawa, M. Ando, T. Ohji, S. Kanzaki, J. Am. Ceram. Soc. 84 (1) (2001) 230. 